\title{
Epilepsy and its Management in Relation to Psychiatry
}

\section{Amir Mufaddel*}

Community Mental Health Services, Behavioural Sciences Institute, Al Ain Hospital, United Arab Emirates University, United Arab Emirates

\begin{abstract}
The relation between psychiatry and epilepsy remains one of the topics that have been continuously attracting attention in medical literature since the time of Hippocrates. Forced normalization was later conceptualized when biological treatment for psychiatric disorders was developed. Recently, several reports were published regarding possible adverse psychiatric effects of antiepileptic medications. The picture is further complicated by the fact that psychotropic medications, which may be used for treatment of psychiatric disorders in epilepsy, can have negative
\end{abstract} effects on seizure control.

This paper aimed to review psychiatric presentations in individuals with epilepsy and the adverse psychiatric effects of antiepileptic medications. The paper also briefly discusses available treatment options with regards of better psychotropic treatment options.

Recent developments in this field led to the observation that psychiatric symptoms, in relation to seizure, can be categorized into the following types pre-ictal, post-ictal, inter-ictal and ictal symptoms. Depression and anxiety are commonly associated with epilepsy and can occur as pre-ictal, ictal, post-ictal or inter-ictal symptoms. Depression can be severe and suicidal behavior has been reported as one of the common psychiatric associations with epilepsy and has also been linked to use of some antiepileptic drugs. Psychosis can occur as an acute or chronic form. Transient pleomorphic postictal psychosis particularly observed in those with hippocampal sclerosis. There is an increased risk of developing schizophrenia and schizophrenia-like psychosis in patients with epilepsy. Psychiatric symptoms can also emerge during use of some of the antiepileptic drugs. Adverse psychiatric events include depression, psychosis, suicidal risk, and cognitive impairment. Some of the psychotropic medications are known to be epileptogenic. Therefore, selection of psychotropic medications should be based on their possible effects on seizures and those known to be epileptogenic should be avoided.

Keywords: Epilepsy; Psychosis; Mood disorders; Anxiety; Psychotropic; Antiepileptic

\section{Introduction}

Epilepsy is a chronic disorder characterized by recurrent seizures, or a seizure of a paroxysmal brain dysfunction due to excessive neuronal discharge. The Greek physician Hippocrates wrote the first book on epilepsy more than 2000 years ago, and he stated that "Most melancholics usually also become epileptics and epileptics melancholics" [1].

The association between psychological symptoms and epilepsy can be related to several factors such as the possibility of shared etiology, stigma and psychosocial factors related to epilepsy, and the adverse psychiatric reactions of antiepileptic drugs. Some of the psychotropic medications are epileptogenic; and this should also be considered when prescribing a psychotropic medication for an epileptic patient. Psychiatric disorders in epilepsy can be classified, based on their relationship to seizures, into the following categories: pre-ictal symptoms (occur before seizure/ prodromal states and mood disturbance), inter-ictal psychiatric disorders occurring between seizures; ictal psychiatric symptoms occurring during the seizure events; and post-ictal psychiatric symptoms following seizures (Table1). Occasionally, an altered mental state can be the only sign of non-convulsive status epilepticus such as that of complex partial or absence types of seizures [2].

Psychiatric comorbidity is common among patients with epilepsy with rates increasing at least two-folds in that attending specialist care. Psychiatric conditions that are commonly associated with epilepsy are depressive disorder, anxiety disorder, attention-deficit hyperactivity disorder and psychoses [3]. For example, a recent study including 319 patients with focal epilepsy suggested that about $58 \%$ of patients with focal epilepsy have either a current or past history of a psychiatric disorder. Depression was found to be the most common psychiatric diagnosis occurring in $32.6 \%$ of patients. Other psychiatric diagnoses were psychotic disorders (7.2\%), anxiety (6.9\%), substance misuse/ dependence $(3.1 \%)$, somatoform disorders $(4.7 \%)$, and personality disorders (13.8\%) [4]. Data from the California Health Interview Survey conducted in 2005, including data from 604 adult participants with history of epilepsy, indicated that $27 \%$ had psychological distress associated with epilepsy, and $84 \%$ of them needed mental health care, but only $57 \%$ have been seen by a mental health professional [5]. A recent population-based cohort study comparing 938 patients whom were newly diagnosed with epilepsy and 518,748 subjects without epilepsy revealed that the incidence of psychiatric disorders for people with epilepsy was 94.1 per 1000 persons-year and for those without epilepsy were 22.6 per 1000 persons-year. The epilepsy cohort had the highest risks in mental retardation bipolar disorder and alcohol or drug induced psychosis. The highest risk occurred in the first year following the diagnosis of epilepsy [6].

Behavioral aspects of epilepsy were well-studied in temporal lobe epilepsy and mesial temporal lobe epilepsy compared with other types of epilepsy such as frontal lobe epilepsy. This may be due to the fact that temporal lobe epilepsy represents the majority of cases with

*Corresponding author: Amir Mufaddel, Community Mental Health Services Behavioural Sciences Institute, United Arab Emirates University, Al Ain Hospital, P.O. Box 1006, United Arab Emirates, Tel: 00971556292529; E-mail: khalifaamir@yahoo.co.uk

Received August 13, 2014; Accepted September 15, 2014; Published September 30, 2014

Citation: Mufaddel A (2014) Epilepsy and its Management in Relation to Psychiatry. Int J Neurorehabilitation 1: 121. doi:10.4172/2376-0281.1000121

Copyright: () 2014 Mufaddel A. This is an open-access article distributed under the terms of the Creative Commons Attribution License, which permits unrestricted use, distribution, and reproduction in any medium, provided the original author and source are credited. 


\begin{tabular}{|c|c|c|}
\hline Pre-ictal symptoms & $\begin{array}{l}\text { Prodromal states that occur several hours or days } \\
\text { before seizures }\end{array}$ & $\begin{array}{l}\text { Increasing tension. } \\
\text { Anxiety. } \\
\text { Depression }\end{array}$ \\
\hline Ictal psychiatric symptoms & $\begin{array}{l}\text { Psychiatric disturbances that are directly related to } \\
\text { seizure. }\end{array}$ & $\begin{array}{l}\text { Confusional states. } \\
\text { Affective symptoms and anxiety. } \\
\text { Automatisms. } \\
\text { Hallucinations (olfactory or gustatory). }\end{array}$ \\
\hline Post-ictal psychiatric symptoms & $\begin{array}{l}\text { Psychiatric disturbances that occur hours following } \\
\text { seizures. }\end{array}$ & $\begin{array}{l}\text { Cognitive symptoms, delirium and impaired. } \\
\text { consciousness } \\
\text { Psychosis. } \\
\text { Violence is rare. }\end{array}$ \\
\hline Inter-ictal psychiatric disorders. & Psychiatric disorders that occur between seizures. & $\begin{array}{l}\text { Cognitive symptoms. } \\
\text { Mood disorders and suicide (depression is } \\
\text { non). } \\
\text { Anxiety. } \\
\text { Inter-ictal psychosis. } \\
\text { Sexual behavior. } \\
\text { Personality changes. } \\
\text { Somatoform and dissociative disorders }\end{array}$ \\
\hline
\end{tabular}

Table 1: Classification of psychological and behavioral disturbances that occur in epilepsy

focal epilepsy, has well-defined underlying pathology (hippocampal sclerosis), is frequently associated with febrile convulsions, has early onset of symptoms, and has prominent neuropsychiatric presentation and memory impairment [7].

This review focuses on the various psychiatric presentations commonly associated with epilepsy. Psychiatric symptoms that emerge as side effects of antiepileptic medications, such as possible risk of depression and suicide, will also be discussed as well as the effects of psychotropic medications on seizures' control and the recommendations for selecting psychotropic medications for treatment in patients with epilepsy.

\section{Mood and Anxiety Disorders}

Depression and anxiety are common findings in patients with epilepsy occurring in $9-37 \%$ and $11-25 \%$ of patients respectively. Higher rates of depression are particularly associated with uncontrolled epilepsy [8].

Symptoms of anxiety are commonly associated with temporal lobe epilepsy; and they can occur as inter-ictal, ictal, or post-ictal anxiety states. Anxiety and fear are the most common form of ictal affect and can be associated with palpitations, rising epigastric sensation, mydriasis, diaphoresis and pallor [1]. A nationally representative population-based study, recently conducted in England, documented that about one-third of patients with epilepsy had anxiety or depressive disorder; and the conditions which were particularly associated with epilepsy included social phobia, agoraphobia, generalized anxiety disorder, depression, and suicidality [9].

The presentation of depression in patients with epilepsy may occur with the same criteria of primary depressive disorders, but may also occur with atypical pleomorphic features [10]. Barry, in his review of mood disorders in epilepsy, identified some risk factors for developing mood disorders in people with epilepsy. These include neurobiological factors, iatrogenic factors (effect of antiepileptic drugs), and psychosocial factors. The depletion of biogenic amines and GABA plays a role in the pathogenesis of both depression and epilepsy. Additionally, frontal lobe dysfunction and decrease in metabolic activity has been recognized in epilepsy as well as in idiopathic depression. Use of antiepileptic drugs may be associated developing symptoms of mood disorders. For example, depression can be induced by barbiturates, topiramate, tiagabine, vigabatrin, and felbamate. Hypomanic states has also been reported with antiepileptic medications such as zonisamide. Psychosocial factors that may predict developing depression in patients with epilepsy include level of social support, stigma, external locus of control, and poor vocational adjustment [11]. The relationship between mood disorders and epilepsy seems to be bidirectional. Patients with epilepsy have increased risk of depression, and patients with depression may also have a risk of developing epilepsy. Both conditions share common pathogenic mechanisms including changes in neurotransmitters such as serotonin, norepinephrine, glutamate, and GABA [10].

Patients with history of depression and epilepsy may have more frequent focal epilepsies arising from the temporal lobe. There was no difference between patients with depression and controls with respect to severity of epilepsy [12].

Mood disorders may also occur following surgery for epilepsy. Therefore, psychiatric follow-up and evaluation is suggested after surgery to avoid complications such as risk of suicide. Study including 38 patients with history of surgery for intractable temporal lobe epilepsy suggested a relation between history of preoperative postictal psychosis and with developing manic or depressive episodes following surgery particularly for those who had left sided lobectomy [13].

Risk of suicide is four times greater than the general population and deliberate self harm is 6 times more frequent in patients with epilepsy particularly in those who have temporal lobe epilepsy and following surgical treatment [2].

\section{Psychosis}

Psychotic symptoms in epilepsy usually occur in the inter-ictal or post-ictal states, and they are rarely occurring in the ictal states. Therefore, psychosis in patients with epilepsy can be divided into three types including chronic and acute interictal psychoses and postictal psychosis. The mentioned types represent about $95 \%$ of psychotic symptoms that occur in patients with epilepsy [14].

Transient postictal psychotic episodes are more common in patients with unilateral hippocampal sclerosis than those who have negative MRI findings [15]. About $88 \%$ of patients presenting with postictal psychosis were found to have a relative increase of right temporal perfusion and SPECT findings suggested a trend of rightsided temporal predominance regardless of the location (right sided / left sided) of the pathology suspected during a non-psychotic state [16]. Psychotic symptoms usually occur 12-72 hours following seizure and can be delayed up to one week. The Postictal psychosis has pleomorphic 
presentation which may include delusions of persecution, grandiosity, and reference. Delusions can also be of somatic and religious types. Catatonia and hallucinations can also occur; and mood symptoms are often prominent [17].

Chronic psychotic disorders such as schizophrenia and related pshoses, should be considered as a possible differential diagnosis for psychotic symptoms in patients with epilepsy. There is an increased risk of schizophrenia and schizophrenia-like psychosis in patients with epilepsy, which increases with age, presence of family history of psychosis, family history of epilepsy and developing epilepsy at a later age. The increased risk for schizophrenia or schizophrenia-like psychosis was not related to gender or type of epilepsy [18]. Patients who have history of Schizophrenia and epilepsy were found to have an earlier onset and more severe forms of epilepsy characterized by history of status epilepticus, and multiple seizure types compared to controls who have epilepsy but no psychiatric symptoms. They have shown various EEGs abnormalities including temporal lobe discharges but there was no lateralization to either side ${ }^{(12)}$.

\section{Aggression}

In relation to seizures, aggression in people with epilepsy can be ictal, post-ictal or inter-ictal, and it is a kind of affective aggression that is usually associated with high emotional arousal, anger or fear [19]. One large series suggest that ictal violence is extremely rare and was found to be less than $0.3 \%$ [20]. Post-ictal aggression is rare but more common than aggression occurring during the seizure episodes. Postictal aggression may be related more too male gender and psychotic experiences and can be recurrent and stereotyped following a cluster of seizures than after a single ictus. Patients are usually remorseful in the inter-ictal period [21].

Aggressive behavior in patients with temporal lobe epilepsy is associated with male gender, early onset of seizures, long-standing behavioral problems, low IQ scores and poor occupational records [22]. Brain structures that mediate aggression include periaqueductal grey matter, hypothalamus, amygdala, limbic structures, and frontal lobes [19,22-24].

Examination of 50 patients with temporal lobe epilepsy of which 25 patients with and 25 without history of intermittent explosive disorder, revealed no higher prevalence of amygdala sclerosis in the aggressive patients. However, $20 \%$ of patients with temporal lobe epilepsy and aggression had severe amygdala atrophy, and $28 \%$ had left temporal lobe lesions affecting the amygadala and periamygdaloid structures [19].

Frontal lobe epilepsy may present with disinhibition, loss of impulse control, and impulsive aggression. Such presentations can be related to the orbitofrontal cortex which connects the frontal and limbic structures which are involved in the control of behavior. Deviant social behavior and affective symptoms can also be associated with damage to the anterior cingulated gyrus which has connections to the amygdala [7].

\section{Cognitive Symptoms and Pervasive Developmental Disorders}

Cognitive symptoms are relatively common in patients suffering from epilepsy, particularly chronic and refractory forms of epilepsy [25]. Study of learning disabilities in children with epilepsy indicated that $48 \%$ had learning disability reflected in at least one academic area by using the IQ-achievement discrepancy definition. By using low-achievement definitions, $41 \%$ to $62 \%$ of children had difficulties in at least one academic area [26]. Progressive cognitive decline has been reported in patients with epilepsy. Factors that can be implicated include the nature, timing, and course of cognitive impairment in addition to factors related to epilepsy such as chronic symptoms, and refractory epilepsy [27].

There is an increased incidence of Attention Deficit Hyperactivity Disorder (ADHD) in patients with epilepsy which is estimated to be about 7.76 cases per 1000 person-years compared with an incidence of 3.22 in patients without history of epilepsy. Similarly, the incidence of epilepsy is greater in patients suffering from ADHD approaching 3.24 cases per 1000 person-year, compared with an incidence of 0.78 in those without history of ADHD [28].

There is a high prevalence of autism in children with epilepsy and there is also a high prevalence of epilepsy in children diagnosed with autism. Both conditions are also associated with intellectual disabilities and this observation suggests that intellectual disability can be a possible connection between the two disorders [29]. Study of 150 individuals who were previously diagnosed with childhood autism indicated that epilepsy developed in $22 \%$ of participants when they were later assessed at the age of 22 years and above. The onset of seizures was after 10 years of age for most of the cases; and they were predominantly generalized tonic-clonic seizures (88\%) [30].

\section{Other Psychiatric Comorbidities}

Example of other psychiatric problems that may exist in patients with epilepsy are the dissociative symptoms which can manifest as psychogenic non-epileptic seizures, personality changes and sexual dysfunction such as decreased libido and loss of sexual interest.

Dissociative symptoms manifesting as seizures were reported in $9 \%$ to $50 \%$ of patients in specialist epilepsy centers [31]. Psychogenic non-epileptic seizures (pseudoseizures) and dissociative states are very difficult to differentiate from epileptic seizures. Video electroencephalogram is the gold standard for proper diagnosis, but this procedure is expensive and time-consuming [32]. Diagnosis of pseudoseizure is suggested by identifiable psychosocial precipitating factors, history of physical or sexual abuse, family history of psychiatric problems, unusual or variable pattern of seizures, and absence of autonomic signs [2].

There is a debate about the presence of certain personality characteristics linked to epilepsy. Hisorically, the epileptic personality has been described by the following characteristics: egocentricity, religiosity, irritability, quarrelsomeness, and sticky thought processes. Later surveys suggest that only minorities of patients with epilepsy (particularly those with temporal lobe lesions) have serious difficulties related to personality [2]. Some studies suggest that personality disorders can also occur in other types of epilepsy and not only temporal lobe epilepsy. For example, one study including 43 patients with juvenile myoclonic epilepsy has shown that personality disorders were present in $23 \%$ of patients [33].

Although reproductive and sexual lives are normal in most of the patients with epilepsy, around $20 \%$ to $30 \%$ of females have dysfunctions in libido, arousal, and orgasm. Sexual problems in males with epilepsy 
including problems of sexual interest and sexual performance which can be related to increased sex hormone-binding globulin levels and lower bioactive testosterone levels due to use of enzyme-inducing antiepileptic medications such as carbamazepine [34].

\section{Psychiatric Side Effects of Antiepileptic Drugs}

Despite the fact that some antiepileptic medications have positive effects in improving psychiatric symptoms ( commonly used as mood stabilizers, such as valproate, carbamazepine, lamotrigine; and some can improve anxiety symptoms), but they can also be associated with adverse psychiatric events including behavioral problems (most commonly), affective symptoms and rarely psychosis [35]. Table 2 summarizes some of the adverse psychiatric events that can be associated with antiepileptic drug treatment. New onset of psychiatric symptoms such as psychosis and depression can occur with anticonvulsant drugs. Paradoxical onset of depression in patients who have well-controlled seizures by antiepileptic drug treatment may be explained by forced normalization and the effect of antiepileptic medications on lowering the levels of folic acid [36].

Few cognitive symptoms can be associated with vigabatrin, topiramate, tiagabine, gabapentin, lamotrigine and levetiracetam [37].

Psychotic symptoms can be induced by ethosuximide, vigabatrin, phenytoin, topiramate carbamazepine, phenobarbital, primidone and benzodiazepines. Psychosis can occur in $6 \%$ of patients of patients receiving topiramate, $0.8 \%$ of patients with tiagabine treatment and $0.3 \%$ of patients taking lamotrigine. Between $2-4 \%$ of patients taking vigabatrin can develop schizophrenia-like psychosis [37-40].

Depression can be associated with the following antiepileptic drugs: acetazoleamide, barbiturates, ethosuximide, carbamazepine, gabapentin, felbamate, levetiracetam, piracetam, phenytoin, tiagabine, topiramate, vigabatrin and zonisamide. There is limited evidence of self-harm and suicidal behavior linked to levetiracetam, tiagabine, topiramate and vigabatrin [41].

In 2008, the U.S. Food and Drug Administration (FDA) published a meta-analysis of data including all clinical trials involving antiepileptic drugs. Suicidal risk was found to be 0.43 per 1000 patients in active drug arms compared with a rate of 0.22 per 1000 in the placebo arm. However, risk of suicide due to antiepleptic drugs remains a controversial issue. Careful psychiatric assessment and screening for depressive symptoms and suicidal thoughts is advisable both before and during treatment with antiepileptic medications [42-44].

\section{Use of Psychotropic Medications for Patients with Epilepsy}

Some of the psychotropic medications are associated with a doserelated risk of reducing seizure threshold, particularly if they are inducing severe hyponatraemia. Therefore, treatment should be started with lower doses and increased gradually [41].

Increased incidence of seizures is associated with antipsychotics particularly clozapine and olanzapine, and with the antidepressant clomipramine. Other psyhotropic medications which have high incidence of seizures include alprazolam, bupropion immediate release form, and quetiapine [45].

Some studies found no statistically significant difference in frequency of seizures between the period before using psychotropics and treatment period. This finding has been documented in a retrospective assessment of 57 consecutive patients with epilepsy who are also using different psychotropic medications indicated that seizure frequency decreased in $33 \%$, was unchanged in $44 \%$, and increased in $23 \%$ of patients during psychotropic drug therapy [46].

Good choices of antipsychotic medications for patients with epilepsy include: trifluperazine, haloperidol, and supliride which have low proconvulsive effects. Care is required when using risperidone, olanzapine (may affect EEG and myoclonic seizures reported), quetiapine (may reduce seizure threshold up to two-fold), and aripirazole. Antipsychotics that are known as being epileptogenic should be avoided such as clozapine, chlorpromazine and zotepine. Good choices among antidepressants include moclobemide, and Selective Serotonin Re-uptake Inhibitors (SSRIs). Mirtazapine, reboxetine, venlafaxine and duloxetine should be used with caution. Bupropion and most of the tricyclic antidepressants (such as amitriptyline and clomiramine) are epileptogenic and should be avoided in patients with epilepsy [41].

\section{Conclusion}

Epilepsy and its treatment are associated with a wide range of psychiatric symptoms including mood disorders, psychosis and cognitive impairment. On the other hand, some of the psychotropic medications used for treatment of emerging psychiatric symptoms in patients with epilepsy may reduce seizure threshold. Evaluation of psychiatric symptoms in relation to seizures is essential for decision regarding the necessity of using psychotropic medications and the choice of psychotropics, if needed, depends on their effect on seizure control.

\begin{tabular}{|c|c|c|}
\hline Depression & $\begin{array}{l}\text { Acetazoleamide, barbiturates, ethosuximide, carbamazepine, } \\
\text { gabapentin, felbamate, levetiracetam, piracetam, phenytoin, } \\
\text { tiagabine, topiramate, vigabatrin and zonisamide. }\end{array}$ & $\begin{array}{l}\text { Etiology may be related to forced normalization or } \\
\text { decreasing folic acid levels. } \\
\text { Increased risk with rapid dose titration (e,g, } \\
\text { topiramate) }\end{array}$ \\
\hline Suicidal behavior & levetiracetam, tiagabine, topiramate and vigabatrin. & Limited evidence \\
\hline Cognitive symptoms & $\begin{array}{l}\text { Vigabatrin, topiramate, tiagabine, gabapentin, lamotrigine and } \\
\text { levetiracetam. }\end{array}$ & implicated Both epilepsy and antiepileptic drugs may be \\
\hline Psychotic symptoms & $\begin{array}{l}\text { Ethosuximide, vigabatrin, phenytoin, topiramate, carbamazepine, } \\
\text { phenobarbital, primidone and benzodiazepines. }\end{array}$ & $\begin{array}{l}\text { May be related to forced normalization (psychotic } \\
\text { symptoms emerge when frequency of seizures diminished). }\end{array}$ \\
\hline
\end{tabular}

Table 2: Adverse Psychiatric Events Associated with Antiepileptic Drugs 


\section{References}

1. Erasmo A, Passaro (2003) Psychiatric Comorbidity in Epilepsy. Primary Psychiatry 10: 72-79.

2. Shorter Oxford Textbook of psychiatry (5th edition). Gelder M, Harrison B Cowen P (Eds). Oxford University Press, oxford, UK. Chapter 14, 346-350.

3. Gaitatzis A, Carroll K, Majeed A, W Sander J (2004) The epidemiology of the comorbidity of epilepsy in the general population. Epilepsia 45: 1613-1622.

4. Adams SJ, O'Brien TJ, Lloyd J, Kilpatrick CJ, Salzberg MR, et al. (2008) Neuropsychiatric morbidity in focal epilepsy. Br J Psychiatry 192: 464-469.

5. Thompson AW, Kobau R, Park R, Grant D (2012) Epilepsy care and mental health care for people with epilepsy: California Health Interview Survey, 2005 Prev Chronic Dis 9: E60.

6. Chang HJ, Liao CC, Hu CJ, Shen WW, Chen TL, et al. (2013) Psychiatric disorders after epilepsy diagnosis: a population-based retrospective cohor study. PLoS One 8: e59999.

7. Helmstaedter C (2001) Behavioral Aspects of Frontal Lobe Epilepsy. Epilepsy Behav 2: 384-395.

8. Kwon OY, Park SP (2014) Depression and anxiety in people with epilepsy. J Clin Neurol 10: 175-188.

9. Rai D, Kerr MP, McManus S, Jordanova V, Lewis G, et al. (2012) Epilepsy and psychiatric comorbidity: a nationally representative population-based study. Epilepsia 53: 1095-1103.

10. Kanner AM (2006) Depression and epilepsy: a new perspective on two closely related disorders. Epilepsy Curr 6: 141-146.

11. Barry JJ (2003) The recognition and management of mood disorders as a comorbidity of epilepsy. Epilepsia 44 Suppl 4: 30-40.

12. Schmitz EB, Robertson MM, Trimble MR (1999) Depression and schizophrenia in epilepsy: social and biological risk factors. Epilepsy Res 35: 59-68.

13. Kanemoto K, Kawasaki J, Mori E (1998) Postictal psychosis as a risk factor for mood disorders after temporal lobe surgery. J Neurol Neurosurg Psychiatry 65: 587-589.

14. Kanemoto K, Tadokoro Y, Oshima T (2012) Psychotic illness in patients with epilepsy. Ther Adv Neurol Disord 5: 321-334.

15. Kanemoto K, Takeuchi J, Kawasaki J, Kawai I (1996) Characteristics of temporal lobe epilepsy with mesial temporal sclerosis, with special reference to psychotic episodes. Neurology 47: 1199-1203.

16. Oshima T, Motooka $H$, Kanemoto $K$ (2011) SPECT findings during postictal psychoses: predominance of relative increase of perfusion in right temporal lobe. Epilepsia 52: 1192-1194.

17. Sachdev PS (2007) Alternating and postictal psychoses: review and a unifying hypothesis. Schizophr Bull 33: 1029-1037.

18. Qin P, Xu H, Laursen TM, Vestergaard M, Mortensen PB (2005) Risk for schizophrenia and schizophrenia-like psychosis among patients with epilepsy: population based cohort study. BMJ 331: 23.

19. van Elst LT, Woermann FG, Lemieux L, Thompson PJ, Trimble MR (2000) Affective aggression in patients with temporal lobe epilepsy: a quantitative MRI study of the amygdala. Brain $123: 234-243$.

20. Delgado-Escueta AV, Mattson RH, King L, Goldensohn ES, Spiegel H, et al. (1981) Special report. The nature of aggression during epileptic seizures. N Engl J Med 305: 711-716.

21. Gerard ME, Spitz MC, Towbin JA, Shantz D (1998) Subacute postictal aggression. Neurology 50: 384-388.

22. Herzberg JL, Fenwick PB (1988) The aetiology of aggression in temporal-lobe epilepsy. Br J Psychiatry 153: 50-55.

23. Brandão ML, Cardoso SH, Melo LL, Motta V, Coimbra NC (1994) Neura substrate of defensive behavior in the midbrain tectum. Neurosci Biobehav Rev 18: $339-346$

24. Raine A, Stoddard J, Bihrle S, Buchsbaum M (1998) Prefrontal glucose deficits in murderers lacking psychosocial deprivation. Neuropsychiatry Neuropsychol Behav Neurol 11: 1-7.
25. Pitkänen A, Sutula TP (2002) Is epilepsy a progressive disorder? Prospects for new therapeutic approaches in temporal-lobe epilepsy. Lancet Neurol 1 : 173-181.

26. Fastenau PS, Jianzhao Shen, Dunn DW, Austin JK (2008) Academic underachievement among children with epilepsy: proportion exceeding psychometric criteria for learning disability and associated risk factors. J Learn Disabil 41: 195-207

27. Hermann B, Seidenberg M (2007) Epilepsy and cognition. Epilepsy Curr 7: 1-6.

28. Chou IC, Chang YT, Chin ZN, Muo CH, Sung FC, et al. (2013) Correlation between epilepsy and attention deficit hyperactivity disorder: a populationbased cohort study. PLoS One 8: e57926.

29. Berg AT, Plioplys S (2012) Epilepsy and autism: is there a special relationship? Epilepsy Behav 23: 193-198.

30. Bolton PF, Carcani-Rathwell I, Hutton J, Goode S, Howlin P, et al. (2011) Epilepsy in autism: features and correlates. Br J Psychiatry 198: 289-294.

31. Francis $P$, Baker GA (1999) Non-epileptic attack disorder (NEAD): a comprehensive review. Seizure 8: 53-61.

32. Ali S, Jabeen S, Arain A, Wassef T, Ibrahim A, at al. (2011) How to Use You Clinical Judgment to Screen for and Diagnose Psychogenic Nonepileptic Seizures without Video Electroencephalogram. Innov Clin Neurosci 8: 36-42.

33. Trinka E, Kienpointner G, Unterberger I, Luef G, Bauer G, et al. (2006) Psychiatric comorbidity in juvenile myoclonic epilepsy. Epilepsia 47: 20862091.

34. Harden CL (2006) Sexuality in men and women with epilepsy. CNS Spectr 11 13-18.

35. Schmitz B (2006) Effects of antiepileptic drugs on mood and behavior. Epilepsia 47 Suppl 2: 28-33.

36. Harden CL, Goldstein MA (2002) Mood disorders in patients with epilepsy: epidemiology and management. CNS Drugs 16: 291-302.

37. Turjanski N, Lloyd GG (2005) Psychiatric side-effects of medications: recent developments. Advances in Psychiatric Treatment: 11: 58-70.

38. Zesiewicz TA, Tullidge A, Tidwell J, Sullivan KL, Hauser RA (2006) Topiramateinduced psychosis in patients with essential tremor: report of 2 cases. Clin Neuropharmacol 29: 168-169.

39. Levinson DF, Devinsky O (1999) Psychiatric adverse events during vigabatrin therapy. Neurology 53: 1503-1511.

40. Kanner AM, Nieto JC (1999) Depressive disorders in epilepsy. Neurology 53 S26-32.

41. Taylor D, Paton C, Kapur S (2009) The south London and Maudsley NHS Foundation Trust and Oxleas NHS Foundation Trust. Prescribing Guidelines (11th edition).Wiley Blackwell, West Sussex, UK.

42. Britton JW, Shih JJ (2010) Antiepileptic drugs and suicidality. Drug Healthc Patient Saf 2: 181-189.

43. Machado RA, Espinosa AG, Melendrez D, González YR, García VF, et al. (2011) Suicidal risk and suicide attempts in people treated with antiepileptic drugs for epilepsy. Seizure 20: 280-284.

44. Mula M, Sander JW (2013) Suicide risk in people with epilepsy taking antiepileptic drugs. Bipolar Disord 15: 622-627.

45. Alper K, Schwartz KA, Kolts RL, Khan A (2007) Seizure incidence in psychopharmacological clinical trials: an analysis of Food and Drug Administration (FDA) summary basis of approval reports. Biol Psychiatry 62 : 345-354.

46. Gross A, Devinsky O, Westbrook LE, Wharton AH, Alper K (2000) Psychotropic medication use in patients with epilepsy: effect on seizure frequency. $J$ Neuropsychiatry Clin Neurosci 12: 458-464. 